\title{
Single Step Purification of a Small Non-mAb Biologic by Peptide-ELP based Affinity Precipitation
}

\author{
Akshat Mullerpatan ${ }^{1}$, Ronit Ghosh ${ }^{1}$, Erin Kane ${ }^{1}$, André Nascimento ${ }^{1}$, Henrik Andersen ${ }^{2}$, \\ Steven Cramer ${ }^{1}$, and Pankaj Karande ${ }^{1}$ \\ ${ }^{1}$ Rensselaer Polytechnic Institute \\ ${ }^{2}$ Bristol-Myers Squibb Co
}

May 6, 2020

\begin{abstract}
Affinity precipitation using stimulus-responsive biopolymers such as Elastin-like Polypeptides (ELPs) have been successfully employed for the purification of monoclonal antibodies. In the current work, we extend these studies to the development of an ELP-peptide fusion for the affinity precipitation of the therapeutically relevant small non-mAb biologic, AdP. A 12-mer affinity peptide ligand (P10) was identified by a primary phage biopanning followed by a secondary in-solution fluorescence polarization screen. Peptide P10 and AdP interacted with a KD of $19.5 \mu \mathrm{M}$. A fusion of P10 with ELP was then shown to be successful in selectively capturing the biologic from a crude mixture. While $\mathrm{pH}$ shifts alone were not sufficient for product elution, the use of $\mathrm{pH}$ in concert with fluid phase modifiers such as $\mathrm{NaCl}$, arginine or ethylene glycol was successful. In particular, the use of pH 8.5 and an arginine concentration of $500 \mathrm{mM}$ enabled $>80 \%$ product recovery. The overall process performance evaluated by SDS-PAGE and reversed-phase UPLC analyses, indicated the successful single-step purification of the biologic from an E. coli lysate resulting in $\sim 90 \%$ purity and $>80 \%$ recovery. These results demonstrate that phage display can be readily employed to identify a peptide ligand capable of successfully carrying out the purification of a non-antibody biological product using ELP-based affinity precipitation.
\end{abstract}

\section{Introduction}

While monoclonal antibodies continue to dominate the biologics market, there is significant activity in the discovery of non-mAb scaffolds as biological products for several reasons such as lower half-life, smaller size and ease of engineering. Out of the different protein scaffold families, one is the $10^{\text {th }}$ Fibronectin Type III (10Fn3) domain, popularly known as 'monobodies' or 'Adnectins' (Koide et al., 2012). These molecules also possess flexible loops similar to the CDRs in antibodies but are nearly 10-fold smaller than monoclonal antibodies ( ${ }^{10}-12 \mathrm{kDa}$ in size compared to monoclonal antibodies which are ${ }^{\sim} 140-150 \mathrm{kDa}$ in size) (Lipovsek, 2011). It is well known that protein A targets the conserved Fc region of IgGs and is a well-established platform affinity ligand for the purification of monoclonal antibodies (Huse et al., 2002). However, unlike IgGs, small scaffold proteins such as Adnectins contain only a single domain. A 70-80\% homology was observed from the sequence alignment of existing Adnectin molecules in the literature. These homologous regions can be potentially targeted for a generalizable purification platform for Adnectins.

Peptides have shown considerable potential as affinity ligands for the purification of biological targets due to several favorable attributes such as structural flexibility, stability, sequence diversity and ease of production. While rational approaches have been used for the design of peptide ligands against protein targets (Chandra et al., 2013), combinatorial display techniques have also resulted in the identification of affinity peptides 
against protein targets. Peptide-based phage display has been widely used for the identification of peptide affinity ligands for drug discovery (Nixon et al., 2014), biosensing (Wu et al., 2011), as well as in the affinity purification of biologics (Kelley et al., 2004). Phage display-aided peptide selection employs a procedure known as biopanning which involves challenging a library of bacteriophage against an immobilized target. Strategic washes, negative selections and multiple biopanning rounds result in the discovery of affinity peptide ligands specific to the target. Peptides have been previously employed for the chromatographic purification of mAbs (Yang et al., 2008) as well as various non-mAb targets such as Factor VIII (Kelley et al., 2004), human growth hormone (Chandra et al., 2019), erythropoietin (Kish et al., 2018), Fab fragments (Nascimento et al., 2019) and viruses (Heldt et al., 2008). The moderate affinities of peptide affinity ligands to their corresponding target result in both effective purification as well as relatively milder conditions for elution and subsequent target recovery.

The class of phase transitioning smart biopolymers - elastin-like polypeptides (ELPs) - has been investigated over the last two decades for the downstream processing of proteins (Yeboah et al., 2016). ELPs are typically composed of multiple repeats of the pentapeptide unit V-P-G-X-G (where guest residue X can be any amino acid except proline) and can undergo precipitation upon the change in stimuli such as temperature and salt concentration (Meyer \& Chilkoti, 1999). Different approaches involving ELP-protein fusion systems have been effectively employed for protein purification (MacEwan et al., 2014). However, these often require proteolytic cleavage or the introduction of self-splicing inteins (Fong et al., 2009) to dissociate the ELPtag from the product, resulting in the requirement of additional purification steps. Therefore, an alternate strategy - affinity precipitation - involving fusions of ELPs and affinity ligands have been employed for the tag-free purification of proteins (Madan et al., 2013). Previous work by Cramer and Chen has successfully demonstrated the purification of monoclonal antibodies from complex mixtures using ELP-Z-based affinity precipitation (Sheth et al., 2013). This process was found to be comparable to protein A purification and also readily scalable when performed with membrane filtration steps (Sheth, Bhut, et al., 2014; Sheth, Jin, et al., 2014). The Cramer and Karande labs have also recently demonstrated the purification of model proteins such as Anti-Flag Antibody M2 and streptavidin using peptide-ELP fusions (Mullerpatan et al., 2020).

In this work, we specifically focused on an industrially relevant small non-mAb scaffold protein, P-Adnectin (AdP). At present the purification of AdP has been carried out using affinity tag fusions (Mitchell et al., 2014) or with a series of chromatographic steps. In the current work, we first employed phage panning to identify peptide affinity ligands against AdP. Lead candidates were then synthesized and their binding behavior to the product was evaluated using fluorescence polarization. A peptide-ELP fusion of the best performing candidate was then produced in $E$. coli and various conditions of stoichiometry and $\mathrm{pH}$ were examined to establish appropriate conditions for binding and precipitation of the AdP. Elution conditions were then established using a combination of $\mathrm{pH}$ and fluid phase modifiers. Finally, the optimized capture and elution conditions were employed in a proof of concept experiment demonstrating the utility of a singlestep affinity precipitation process that was capable of capturing $~ 80 \%$ of AdP from a crude $E$. coli lysate at a purity of $\sim 90 \%$.

\section{Materials and Methods}

\section{Materials}

P-Adnectin (AdP) was provided by Bristol-Myers Squibb. Amiconß Ultra-15 centrifugal filter units (NMWL/MWCO of 30 and $100 \mathrm{kDa}$ ) were purchased from Merck Millipore (Darmstadt, Germany). Citric acid, tribasic sodium citrate dihydrate, Tris-HCl, Trizma base, sodium hydroxide, L-cysteine, sodium phosphate (monobasic and dibasic), sodium sulfate, triisopropyl silane (TIPS), trifluoroacetic acid (TFA), dichloromethane (DCM), were purchased from Sigma Aldrich (St. Louis, MO/USA). Dimethylformamide (DMF), piperidine, N-methyl pyrrolidone (NMP), activator 2-(1H -benzotriazol-1-yl)-1,1,3,3-tetramethyluronium hexafluorophosphate (HBTU) and methyl-tertiary-butyl ether (MTBE) were purchased from AGTC Bioprod- 
ucts (Wilmington, MA). Nova-PEG Rink Amide Resin was purchased EMD MilliporeSigma (Burlington, MA). All 20-fluorenylmethoxy carbonyl (Fmoc) amino acids were purchased from 21st Century Biochemicals (Marlborough, MA). Fmoc-Lys-FAM-OH was obtained from AAT Bioquest (Sunnyvale, CA). 10X SDS PAGE running buffer, Kaleidoscope Precision-Plus protein gel standard ladder, Bio-Rad AnykD polyacrylamide gel and SDS-PAGE system were procured from Bio-Rad Laboratories. Yeast extract was obtained from Amresco, while Bacto-tryptone, Bacto-agar were obtained from BD.

Single stranded oligonucleotide sequences and gBlock gene fragments encoding the peptide/linker sequences were purchased from Integrated DNA Technologies (IDT). Pfu Ultra II Hotstart PCR Master Mix for cloning was obtained from Agilent Technologies. NEB PhD-12 Phage Display Library Kit, DH5 $\alpha$ variant NEB5 $\alpha$ cells, DpnI, BamHI, EcoRI, NdeI and PstI restriction enzymes were purchased from New England Biolabs (Ipswich, MA). QiaPrep spin Miniprep kit was ordered from Qiagen (Frederick, MD). Bio-Rad Laboratories MyCycler was used for carrying out PCR. Solid black 384 well plates (CAT 3575) from Corning were used for fluorescence polarization assays. BEH C4 RPLC and BEH 200A SEC columns were obtained from Waters (Milford, MA).

Equipment: Bruker MALDI-Tof II equipment was used for mass spectrometry of the different protein components used in experiments. Intavis Multipep RS instrument was utilized for solid phase peptide synthesis. A Waters Acquity ultra performance liquid chromatography system was utilized for the UPLC analysis of experimental samples.

\section{Phage Biopanning}

One well in a 96 well plate was incubated for 12 hours with the target AdP in PBS pH 7.4 at $4^{0} \mathrm{C}$. Following physisorption, the well was blocked with bovine serum albumin (BSA) for 2 hours at $4^{0} \mathrm{C}$. Any excess unbound BSA was washed away using PBS pH 7.4. A $10 \mu$ l aliquot of the NEB Ph.D.-12 phage display peptide library containing approximately $10^{11}$ plaque forming units (pfu) was then mixed into $140 \mu \mathrm{l}$ PBS $\mathrm{pH} 7.4$ in a $0.5 \mathrm{~mL}$ centrifuge tube and added to the well. All steps of incubation, washing and elution were carried out according to the protocol detailed in the NEB phage display manual at room temperature $\left(23^{0} \mathrm{C}\right) .10$ washes comprising combinations of PBS and PBST (PBS $+0.1 \%$ Tween-20) were performed to remove weakly bound phages from the immobilized AdP. Following the washes, elution was carried out using $150 \mu \mathrm{l} 0.2 \mathrm{M}$ Glycine $\mathrm{pH} 2.2$ for 15 minutes on a rotating platform. The eluate was immediately neutralized by adding $1 \mathrm{M}$ Tris $\mathrm{pH}$ 9.1. Titering of the eluate followed by amplification was carried out according to the NEB phage display manual. This procedure constitutes a single round of biopanning.

The amplified eluate was diluted approximately to $10^{11} \mathrm{pfu}$ and two additional rounds of biopanning were performed. In the subsequent biopanning rounds, stringency of washing was increased by using PBST with a higher concentration of Tween-20 (0.5\%). The final eluate obtained at the end of the third round of biopanning was not further amplified. The unamplified final eluate was titered and 20 unique distinct phage plaques were picked, amplified and the phage supernatants were submitted for sequencing. Sequencing was performed by Genewiz (South Plainfield, NJ) using the -96 gIII sequencing primer $5{ }^{\prime}{ }^{\mathrm{HO}} \mathrm{CCC}$ TCA TAG TTA GCG TAA CG $-3^{\prime}$ and sequences were analyzed using Expasy Translate (https://web.expasy.org/translate/). Figure 1 presents a schematic of the phage panning approach used to identify peptide ligands for AdP.

\section{Peptide Synthesis}

Peptides were synthesized with a C-terminal 5(6)-carboxyfluorescein-labeled lysine, by solid phase peptide synthesis (SPPS) on an Intavis AG Multipep RS (Chicago, IL). Fluorenylmethoxycarbonyl (Fmoc) chemistry

was used to synthesize the peptides (Chandra et al., 2013). Peptide purity was determined by a Waters Acquity ultra-performance liquid chromatography (UPLC) system on a Waters BEH C18 reversed phase liquid chromatography (RPLC) column. 


\section{Fluorescence Polarization}

Stock solutions of labeled peptides were prepared at a concentration of $500 \mathrm{nM}$. Protein solutions of a wide range of concentration $(0.5 \mu \mathrm{M}-200 \mu \mathrm{M})$ were prepared. Typically, 12 protein dilutions were prepared and the change in fluorescence polarization (FP) signal was recorded. $20 \mu$ l of labeled peptide was incubated with $20 \mu \mathrm{l}$ of each protein solution in 384-well black plates. Each peptide-protein concentration pair was analyzed in duplicate. Peptide and protein were incubated with shaking for 3 hours at room temperature, following which the FP values were recorded in a Biotek Synergy HT plate reader. Excitation was carried out using a $485 \pm 20 \mathrm{~nm}$ excitation filter and emission was controlled by a $520 \pm 20 \mathrm{~nm}$ filter, $20 \mathrm{~nm}$ being the spectral bandwidth of each filter. Gain and top probe vertical offset were appropriately modified to obtain analyzable intensity values. Fluorescence polarization values obtained from the experiment were then fitted to a 4parameter logistic equation (Equation 1) to obtain binding affinity ('C' parameter) (Gadagkar \& Call, 2015). Normalized FP change was calculated as $\left(\mathrm{FP}-\mathrm{FP}_{0}\right) / \mathrm{FP}_{0}$, where the $\mathrm{FP}$ is the fluorescence polarization value at a given concentration while $\mathrm{FP}_{0}$ is the base polarization value (at zero target concentration).

$$
Y=\frac{(A-D)}{\left(1+\left(\frac{X}{C}\right)^{B}\right)}+D
$$

Equation 1: 4 parameter logistic curve equation used to fit binding curve data. $\mathrm{A}=$ response at zero analyte concentration, $\mathrm{B}=$ slope factor, $\mathrm{C}=$ inflection point, $\mathrm{D}=$ response at infinite analyte concentration

\section{Peptide-ELP Cloning, Expression and Purification}

Site-directed mutagenesis using Pfu Ultra Hotstart 2x PCR Master Mix, was used for the creation of peptideELP constructs. For the P10 lead peptide, two sets of completely overlapping forward and reverse primers were designed to sequentially clone in one half of the peptide first, followed by the remaining half. The PCR reaction recipe comprised: $1 \mu \mathrm{l}$ template DNA $\left({ }^{\sim} 50-100 \mathrm{ng} / \mu \mathrm{l}\right), 1 \mu \mathrm{l}$ of each primer (to a final concentration of $0.5 \mu \mathrm{M}$ ), $25 \mu \mathrm{l}$ of PfuUltra II Hotstart PCR Master Mix, $2.5 \mu \mathrm{l}$ of DMSO (if required to reduce the melting temperature of primers) and the remaining volume of ultrapure water to make up the reaction volume to 50 $\mu$ l. Denaturation was carried out at $95^{\circ} \mathrm{C}$ for 30 seconds, followed by an annealing step for 20 seconds. The annealing temperature was set depending on the melting temperatures $\left(\mathrm{T}_{\mathrm{m}}\right)$ of the primers. For primers with $\mathrm{T}_{\mathrm{m}}>72^{\circ} \mathrm{C}$, the annealing temperature was kept between 68 and $72^{\circ} \mathrm{C}$, whereas for primers with lower $\mathrm{T}_{\mathrm{m}}$ values, annealing temperatures between $58-62^{\circ} \mathrm{C}$ were used. Following the PCR, DpnI digestion was conducted to degrade parental template DNA for 1 hour at $37^{\circ} \mathrm{C}$. The resulting DNA was transformed into NEB5 $\alpha$ cells using thermal shock. Transformant colonies were picked from the $50 \mu \mathrm{g} / \mathrm{mL}$ Kanamycin plates and grown overnight in LB media. DNA was extracted from the grown cultures by minipreparation using the QIAPrep Spin Miniprep Kit. Sequences were analyzed for correctness using Expasy Translate.

The correctly identified DNA sequences were transformed into BL21DE3. Transformant colonies were picked and grown in $5 \mathrm{~mL} \mathrm{LB}$ media cultures in the presence of $50 \mu \mathrm{g} / \mathrm{mL}$ Kanamycin before addition to $250 \mathrm{~mL}$ Terrific Broth (TB) containing $50 \mu \mathrm{g} / \mathrm{mL}$ Kanamycin. Two routes of expression were tested. The first involved induction with IPTG at $\mathrm{OD}_{600}$ of 0.8-1.0 (1 mM final concentration) and the second was performed in the absence of IPTG. Both routes delivered significantly high expression yields. In the case of IPTG induction, the $250 \mathrm{~mL}$ culture was grown at $37^{0} \mathrm{C}$ for $4-5$ hours until it approached an $\mathrm{OD}_{600}$ value of 0.8 1.0. IPTG was added to a final concentration of $1 \mathrm{mM}$ and the temperature was reduced to $22^{0} \mathrm{C}$ to allow for protein expression to occur. For the expression involving no IPTG addition, the culture was grown for 16-20 hours at a constant temperature of $37^{0} \mathrm{C}$. The culture was then centrifuged at $4000 \mathrm{~g}$ for 20 minutes and the cells were pelleted. The cell pellet was re-suspended in $10 \mathrm{~mL} 1 \mathrm{X}$ PBS with protease inhibitor cocktail and subjected to sonication (in pulses of 5 seconds ON, 5 seconds OFF) for 10 minutes. The lysed cells were centrifuged for 15 minutes at $15,000 \mathrm{~g}$ and the pellet was discarded. Inverse transition cycling, used in previous ELP purification protocols (Sheth et al., 2013) was also employed here to purify the ELP 
constructs. The ELP containing supernatant was isolated and $4 \mathrm{~mL}$ of $1 \mathrm{M} \mathrm{Na}_{2} \mathrm{SO}_{4}$ was added to precipitate the ELP. Centrifugation was performed at $37^{0} \mathrm{C}$ at $15,000 \mathrm{~g}$ for 15 minutes to pellet the precipitated ELP and the impure supernatant was discarded. The pellet containing ELP was then resolubilized in cold PBS $\left(4^{0} \mathrm{C}\right)$ and the solution was centrifuged at $4^{0} \mathrm{C}$ at $15,000 \mathrm{~g}$ for 15 minutes to remove any insoluble impurities. This cycle was repeated at least once more in order to obtain considerably pure ELP construct. Purity of the ELP construct was determined by $\mathrm{C}_{4}$-RP-UPLC analysis and used for further experiments.

\section{Pure Component AdP Precipitation Experiments}

A schematic of the ELP-peptide affinity precipitation process is presented in Figure SI-1. Preliminary binding screening experiments and elution tests were performed in conical $1.5 \mathrm{~mL}$ Eppendorf tubes. Elution screening experiments involving mobile phase modifiers were performed using 'V'-bottomed 96-well plates, in order to increase the throughput of the experiments. In all cases, concentration of AdP used was 40 $\mu \mathrm{M}(1 \mathrm{mg} / \mathrm{mL})$. Also, in all cases, $50 \mu \mathrm{L}$ of $40 \mu \mathrm{M}$ AdP was mixed with $50 \mu \mathrm{L}$ of peptide-ELP construct (at various molar ratios) and incubated for 20 min. $50 \mu \mathrm{L}$ of $1 \mathrm{M} \mathrm{Na}_{2} \mathrm{SO}_{4}$ (final $\mathrm{Na}_{2} \mathrm{SO}_{4}$ concentration of $0.33 \mathrm{M}$ ) was then added to the mixture to precipitate the peptide-ELP-AdP complex. Centrifugation was carried out at $3200 \mathrm{~g}$ for plate-based experiments (10000g for tube-based experiments) for 15 minutes to ensure complete precipitation and pelleting of the complex. $145 \mu \mathrm{L}$ of the supernatant was cleared and recorded as 'S1' or the first supernatant, which accounted for the unbound AdP. Experiments for the quantification of only the pulldown of AdP into the precipitate form were stopped at this point and the amount of AdP in the supernatant was used to measure percentage pulled down. At this point, absence of any $\mathrm{AdP}$ in the supernatant would indicate complete pulldown. In this case, the precipitate (denoted as P1) was re-solubilized in PBS pH 7.4 and subjected to analysis.

In the case of elution screening experiments, the precipitated pellet was then re-solubilized in $100 \mu \mathrm{L}$ of various elution buffers tested. Following re-solubilization of precipitate, $50 \mu \mathrm{L}$ of $1 \mathrm{M} \mathrm{Na}_{2} \mathrm{SO}_{4}$ prepared in the elution buffer was added in order to precipitate the peptide-ELP construct. At this point, complete elution would result in precipitation of only the peptide-ELP, leaving only the AdP in the supernatant (S2, or the second supernatant). However, incomplete elution would result in the precipitation of peptide-ELP as well as any undissociated peptide-ELP-AdP complex upon $\mathrm{Na}_{2} \mathrm{SO}_{4}$ addition. Centrifugation was performed at $3200 \mathrm{~g}$ for plate-based experiments $\left(10000 \mathrm{~g}\right.$ for tube-based experiments) for 15 minutes at $37^{0} \mathrm{C}$ to pellet the precipitate. $145 \mu \mathrm{L}$ of the supernatant S2 was cleared and the pellet, or second precipitate (denoted as P2), was re-solubilized in $150 \mu \mathrm{L}$ PBS at $\mathrm{pH}$ 7.4. The S1, S2 and P2 fractions were analyzed for each experiment using a reversed phase UPLC assay.

\section{Purification of AdP from Crude Mixtures}

Pure AdP was spiked into E. coli lysate to a final concentration of $40 \mu \mathrm{M}$. The AdP-spiked crude mixture was then adjusted to $\mathrm{pH} 4$ which was was identified as the optimal binding condition. Pprecipitate formed during this $\mathrm{pH}$ adjust (containing process related impurities) was removed by centrifugation and the supernatant was collected. $50 \mu \mathrm{L}$ of the peptide-ELP construct was then added to $50 \mu \mathrm{L}$ of the supernatant and incubated for 20 minutes at $23^{0}$ C. $50 \mu \mathrm{L}$ of $1 \mathrm{M} \mathrm{Na}_{2} \mathrm{SO}_{4}$ in binding buffer was added to the AdP-peptide-ELP mixture and centrifuged for $15 \mathrm{~min}$ at $10000 \mathrm{~g}$. The supernatant $\mathrm{S} 1$ was discarded and the pellet was washed three times with $150 \mu \mathrm{L} 0.33 \mathrm{M} \mathrm{Na}_{2} \mathrm{SO}_{4}$ in binding buffer. The precipitate was then re-solubilized and eluted in $200 \mu \mathrm{L}$ of elution buffer (50 mM Tris, $\mathrm{pH} 8.5$ with $500 \mathrm{mM}$ arginine). $100 \mu \mathrm{L}$ of $1 \mathrm{M} \mathrm{Na}_{2} \mathrm{SO}_{4}$ in elution buffer was added to the solution and centrifuged at $10000 \mathrm{~g}$ for $15 \mathrm{~min}$ to pellet the precipitate containing

only dissociated peptide-ELP construct. The purified AdP supernatant S2 was removed and all the fractions of the experiment were evaluated for extent of purification and recovery using UPLC. 


\section{$\mathrm{C}_{4}$ Reversed Phase UPLC Analysis}

An Acquity UPLC Protein BEH C4 column was used for the analysis of all fractions obtained from the binding, precipitation and elution experiments. Linear gradients were carried out at $0.4 \mathrm{ml} / \mathrm{min}$ using two buffers: $5 \%$ acetonitrile, $0.1 \%$ trifluoroacetic acid in water (Buffer A) and 95\% acetonitrile, $0.1 \%$ trifluoroacetic acid in water (Buffer B); all \% in v/v. The gradient consisted of a 0-30\% B gradient in 1.2 column volumes, followed by a 30-70\% B gradient in 3 column volumes and a $70-100 \%$ B gradient in 1.2 column volumes. A regeneration using $100 \% \mathrm{~B}$ was then performed for 1.6 column volumes, followed by a column re-equilibration step at $0 \% \mathrm{~B}$ for 1.6 column volumes. The column effluent was monitored at 280 $\mathrm{nM}$ to quantify the amounts of AdP and peptide-ELP present in the fractions.

\section{SDS PAGE Analysis}

Samples were analyzed for purity using SDS PAGE. $25 \mu \mathrm{L}$ of each sample was mixed with $25 \mu \mathrm{L}$ Laemmli sample buffer and boiled at $95^{\circ} \mathrm{C}$ for 5 minutes. A protein ladder was injected in the first well of a Bio-Rad AnyKd polyacrylamide gel. $25 \mu \mathrm{L}$ of prepared samples were injected into the wells and the gel was run in a Bio-Rad mini-PROTEAN Tetra Cell system in the presence of running buffer (1X Tris/Glycine/SDS buffer). The gels were washed with water and then stained using Coomassie-Blue for 45 minutes, followed by de-staining using deionized water.

\section{Results and Discussion}

\section{Phage Biopanning-based Discovery of Peptide Affinity Ligands for AdP}

The commercially available NEB PhD-12 phage display library was chosen for phage biopanning to identify peptide candidates that bind to AdP. As described in the methods section 2.2, three rounds of phage biopanning (Figure 1) were performed against AdP. Once the biopanning rounds were completed, 20 distinct phage clones from the final eluate were randomly picked and sequenced.

The sequencing yielded 10 unique peptides with multiple repeats obtained for two of the peptides (Table 1).

\section{Fluorescence Polarization Screening for Peptide Ligands}

The 10 lead candidates were then synthesized with a 5(6)-FAM fluorescence label and fluorescence polarization $(\mathrm{FP})$ was carried out to measure the dissociation constants $\left(\mathrm{K}_{\mathrm{d}}\right)$ of their interactions with AdP. The experiments yielded binding curves (Figure 2), which were fitted to a 4-parameter logistic equation. Table 2 lists the $\mathrm{K}_{\mathrm{d}}$ values obtained after fitting for 6 out of the 10 peptides, which showed an appreciable increase in observable signal with increase in AdP concentration. Peptides P3, P5, P6 and P8 were either too impure (as determined by $\mathrm{C}_{18}$-RP-UPLC) or showed a very low change in signal with increase in AdP concentration. From the binding curves shown in Figure 2, out of the 6 peptides, P10 exhibited the strongest binding to AdP.

Appropriate control experiments performed with free 5(6)-FAM dye in solution incubated with AdP indicated no non-specific binding between the dye and AdP. The P10 peptide was also tested against AdI (a different Adnectin provided by Bristol-Myers Squibb) and minimal binding was observed $\left(\mathrm{K}_{\mathrm{d}}>100 \mu \mathrm{M}\right)$ indicating that P10 was indeed specific to AdP.

Since P10 exhibited the lowest $K_{d}$, and hence the strongest binding $\left(K_{d}=19.5 \mu M\right)$, it was carried forward for the development of a peptide-ELP based affinity precipitation process. 


\title{
Development of Peptide-ELP-based Purification of AdP
}

\author{
Binding and Capture of AdP using P10-ELP
}

Initial binding experiments between P10-ELP and AdP were performed over a range of molar ratios of P10ELP to AdP at pH 7.4. It can be observed in Figure 3 that at lower ratios, the capture of AdP increased linearly with the ratio, but the amount of AdP captured saturated at higher ratios, plateauing at a maximum of $85 \%$. This could be due to the moderate affinity of the peptide-AdP interaction, which precludes $100 \%$ binding.

In order to improve the binding of P10-ELP to AdP, the effect of $\mathrm{pH}$ was examined, using $\mathrm{pH}$ conditions of 3.0, 4.0 and 5.0 (Figure 4a). P10-ELP was incubated with AdP (at a ratio of 2:1 of P10-ELP to AdP) and precipitated at these conditions. The results were compared with those obtained at pH 7.4 (Figure 3) demonstrating that the capture improved by nearly a factor of 2 with $\mathrm{pH} 4.0$ resulting in the highest capture of AdP in the precipitate. Since pH 4.0 and 5.0 are close to the pI of AdP, these conditions could result in precipitation of AdP. However, controls performed with $0.33 \mathrm{M}$ sodium sulfate at $\mathrm{pH} 4.0$ and 5.0 with 40 $\mu \mathrm{M} \operatorname{AdP}(\sim 1 \mathrm{mg} / \mathrm{ml})$ resulted in no product precipitation. Binding and capture experiments were repeated at $\mathrm{pH} 4.0$ to examine the impact of molar ratios of P10-ELP to AdP. As can be seen in Figure 4b, while a similar trend was observed for the effect of molar ratio on product capture, a higher capture was obtained at $\mathrm{pH} 4.0$ for all ratios, plateauing at approximately $94 \%$ at 8:1.

\section{Elution of AdP from P10-ELP}

A range of $\mathrm{pH}$ conditions were evaluated for their efficacy at eluting AdP from the P10-ELP affinity reagent. The results indicated that while low $\mathrm{pH}$ did not result in any elution, higher $\mathrm{pH}$ values were able to elute up to $40 \%$ of the AdP. In order to improve the efficacy of the elution step, mobile phase modifiers were included in the eluent. Sodium chloride, ethylene glycol and arginine were selected due to their different mechanisms of disrupting protein-ligand interactions. Sodium chloride is widely employed in bioseparation techniques such as ion exchange and affinity chromatography (Lee \& Chen, 2001) and operates via charge shielding and ion binding to reduce electrostatic interactions (Tsumoto et al., 2007). Ethylene glycol has been shown to weaken hydrophobic interactions while also increasing electrostatic interactions (Hou and Cramer, 2011). In fact, Kelley and co-workers have used ethylene glycol as an eluent in peptide-based affinity purification of Factor VIII (Kelley et al., 2004). Arginine is widely used as an elution modifier for several chromatographic systems. Although the mechanism of interaction of arginine is not fully understood, molecular dynamics simulations (Shukla et al., 2011) and experiments (Hirano et al., 2014) have suggested that arginine interacts with both aromatic as well as positively and negatively charged moieties leading to the mediation of both electrostatic and hydrophobic interactions. Applications have included both multimodal (Holstein et al., 2011) as well as protein A systems (Ejima et al., 2005).

The use of elution buffers with modifiers in our affinity precipitation system requires that the P10-ELPAdP complex remain soluble during elution while also enabling subsequent precipitation of the P10-ELP upon addition of sodium sulfate. The three modifiers employed in this study were examined under elution conditions and were shown to not have any impact on these two critical constraints. Further, since the AdP product was known to be susceptible to dimerization at higher $\mathrm{pH}$, these elution studies were carried out in the presence of $5 \mathrm{mM}$ TCEP (a mild reducing agent to prevent cysteine dimerization).

Figure 5a presents the effects of the three modifier solutions tested independently over a pH range of 3-9. The general trend observed was that high $\mathrm{pH}$ resulted in increased elution, irrespective of which additive was used. The order of modifier effectiveness was arginine $>$ ethylene glycol $>$ sodium chloride. In addition, distinct trends were seen with each modifier as a function of $\mathrm{pH}$. While recovery with sodium chloride exhibited a linear increase in recovery with $\mathrm{pH}$, elution with ethylene glycol was only moderately impacted by $\mathrm{pH}$. In contrast, arginine was very effective as an eluent modifier; with the recovery showing an initial increase followed by saturation as a function of $\mathrm{pH}$. 
Once arginine was identified as the most effective modifier, the effect of concentration was evaluated in the $\mathrm{pH}$ range of 6.0-9.0 where good recoveries were observed at $1 \mathrm{M}$. The effect of concentration was less pronounced at higher $\mathrm{pH}$ (Figure 5b). As can be seen, recoveries of greater than $80 \%$ were attained for conditions with $\mathrm{pH}$ [?] 8.0 and arginine concentrations [?] $300 \mathrm{mM}$. Based on this data, a $\mathrm{pH}$ of 8.5 and an arginine concentration of $500 \mathrm{mM}$ were selected as elution conditions for the complex feed study described below.

\section{Purification of AdP from Crude Mixtures}

After establishing the operating conditions for the affinity precipitation process using pure AdP, we then evaluated the performance of this process with a crude feed, now incorporating three intermediate precipitate wash steps. Importantly, this process worked extremely well with the crude feed, resulting in a final AdP purity of $88 \%$ and an overall recovery of $81 \%$. Figures 6 and 7 show the efficacy of this process using SDS PAGE and RPLC analysis, respectively. As can be seen in the gel, while the feed containing the AdP was complex (lane 5), the final supernatant from the process (lane 8) was quite pure. The RPLC analysis also clearly demonstrates that the final product is significantly purified during the process.

\section{Conclusions}

In this paper, we have successfully developed an ELP-peptide fusion for the affinity precipitation of the therapeutically relevant small non-mAb biologic, AdP. Phage biopanning was first used to identify lead peptides which were then synthesized and evaluated for their binding to the target using fluorescence polarization. A fusion of the top lead peptide with ELP was then produced in E. coli and evaluated for its efficacy to both capture and elute the bioproduct. While binding was favored at low $\mathrm{pH}$ conditions, higher $\mathrm{pH}$ values were more useful for elution. In addition, the use of fluid phase modifiers, in particular arginine, were shown to further improve the elution behavior. Evaluation of the ELP-peptide with a crude mixture demonstrated that both high recovery $(83 \%)$ and purity $(88 \%)$ could be achieved using this affinity precipitation process. While this proof of concept study was successful, further mutagenesis studies could be carried out to improve both the binding and elution behavior of the peptide. This work establishes that peptides identified from phage display can be successfully employed in an ELP fusion format for the purification of a small therapeutic biologic by affinity precipitation. The results and approaches presented in this work can now be extended to other biologics for which there currently do not exist efficient affinity capture processes.

\section{Acknowledgements}

The authors would like to thank Prof. Mattheos Koffas' lab for usage of the plate reader. The authors also acknowledge Bristol-Myers Squibb for providing P-Adnectin, and Prof. Wilfred Chen for providing the ELP plasmid. This work was supported by grant CBET 1403697 from the National Science Foundation. The authors declare no conflict of interest.

\section{References}

Chandra, D., Morrison, C. J., Woo, J., Cramer, S., \& Karande, P. (2013). Design of peptide affinity ligands for S-protein: A comparison of combinatorial and de novo design strategies. Molecular Diversity , 17 (2), 357-369. https://doi.org/10.1007/s11030-013-9436-z

Chandra, D., Timmick, S., Goodwine, C., Vecchiarello, N., Shastry, D. G., Mullerpatan, A., Trasatti, J. P., Cramer, S., \& Karande, P. (2019). Design of peptide ligands for affinity purification of human growth hormone. Journal of Chemical Technology \& Biotechnology . https://doi.org/10.1002/jctb.6029 
Ejima, D., Yumioka, R., Tsumoto, K., \& Arakawa, T. (2005). Effective elution of antibodies by arginine and arginine derivatives in affinity column chromatography. Analytical Biochemistry , 345 (2), 250-257. https://doi.org/10.1016/j.ab.2005.07.004

Fong, B. A., Wu, W.-Y., \& Wood, D. W. (2009). Optimization of ELP-intein mediated protein purification by salt substitution.Protein Expression and Purification , 66 (2), 198-202. https://doi.org/10.1016/j.pep.2009.03.009

Gadagkar, S. R., \& Call, G. B. (2015). Computational tools for fitting the Hill equation to dose-response curves. Journal of Pharmacological and Toxicological Methods , 71 , 68-76. https://doi.org/10.1016/j.vascn.2014.08.006

Heldt, C. L., Gurgel, P. V., Jaykus, L.-A., \& Carbonell, R. G. (2008). Identification of Trimeric Peptides That Bind Porcine Parvovirus from Mixtures Containing Human Blood Plasma. Biotechnology Progress ,24 (3), 554-560. https://doi.org/10.1021/bp070412c

Holstein, M. A., Parimal, S., McCallum, S. A., \& Cramer, S. M. (2012). Mobile phase modifier effects in multimodal cation exchange chromatography. Biotechnology and Bioengineering , 109 (1), 176-186. https://doi.org/10.1002/bit.23318

Hou, Y., \& Cramer, S. M. (2011). Evaluation of selectivity in multimodal anion exchange systems: A priori prediction of protein retention and examination of mobile phase modifier effects. Journal of Chromatography A , 1218 (43), 7813-7820. https://doi.org/10.1016/j.chroma.2011.08.080

Hirano, A., Arakawa, T., \& Kameda, T. (2014). Interaction of arginine with Capto MMC in multimodal chromatography. Journal of Chromatography A , 1338 , 58-66. https://doi.org/10.1016/j.chroma.2014.02.053

Huse, K., Böhme, H.-J., \& Scholz, G. H. (2002). Purification of antibodies by affinity chromatography. Journal of Biochemical and Biophysical Methods , 51 (3), 217-231. https://doi.org/10.1016/S0165-022X(02)00017-9

Kelley, B. D., Tannatt, M., Magnusson, R., Hagelberg, S., \& Booth, J. (2004). Development and validation of an affinity chromatography step using a peptide ligand for cGMP production of factor VIII.Biotechnology and Bioengineering , 87 (3), 400-412. https://doi.org/10.1002/bit.20124

Kish, W. S., Roach, M. K., Sachi, H., Naik, A. D., Menegatti, S., \& Carbonell, R. G. (2018). Purification of human erythropoietin by affinity chromatography using cyclic peptide ligands. Journal of Chromatography B , 1085 , 1-12. https://doi.org/10.1016/j.jchromb.2018.03.039

Koide, S., Koide, A., \& Lipovšek, D. (2012). Target-Binding Proteins Based on the 10th Human Fibronectin Type III Domain (10Fn3). InMethods in Enzymology (Vol. 503, pp. 135-156). Elsevier. http://linkinghub.elsevier.com/retrieve/pii/B9780123969620000069

Lee, W.-C., \& Chen, C.-H. (2001). Predicting the elution behavior of proteins in affinity chromatography on non-porous particles. Journal of Biochemical and Biophysical Methods , 49 (1-3), 63-82. https://doi.org/10.1016/S0165-022X(01)00189-0

Lipovsek, D. (2011). Adnectins: Engineered target-binding protein therapeutics. Protein Engineering Design and Selection ,24 (1-2), 3-9. https://doi.org/10.1093/protein/gzq097

MacEwan, S. R., Hassouneh, W., \& Chilkoti, A. (2014). Non-chromatographic purification of recombinant elastin-like polypeptides and their fusions with peptides and proteins from Escherichia coli. Journal of Visualized Experiments: JoVE ,88 . https://doi.org/10.3791/51583

Madan, B., Chaudhary, G., Cramer, S. M., \& Chen, W. (2013). ELP-z and ELP-zz capturing scaffolds for the purification of immunoglobulins by affinity precipitation. Journal of Biotechnology , 163 (1), 10-16. https://doi.org/10.1016/j.jbiotec.2012.10.007

Meyer, D. E., \& Chilkoti, A. (1999). Purification of recombinant proteins by fusion with thermally-responsive polypeptides. Nature Biotechnology , 17 (11), 1112-1115. https://doi.org/10.1038/15100 
Mitchell, T., Chao, G., Sitkoff, D., Lo, F., Monshizadegan, H., Meyers, D., Low, S., Russo, K., DiBella, R., Denhez, F., Gao, M., Myers, J., Duke, G., Witmer, M., Miao, B., Ho, S. P., Khan, J., \& Parker, R. A. (2014). Pharmacologic Profile of the Adnectin BMS-962476, a Small Protein Biologic Alternative to PCSK9 Antibodies for Low-Density Lipoprotein Lowering. Journal of Pharmacology and Experimental Therapeutics , 350 (2), 412-424. https://doi.org/10.1124/jpet.114.214221

Mullerpatan, A., Chandra, D., Kane, E., Karande, P., \& Cramer, S. (2020). Purification of proteins using peptide-ELP based affinity precipitation. Journal of Biotechnology , 309 , 59-67. https://doi.org/10.1016/j.jbiotec.2019.12.012

Nascimento, A., Mullerpatan, A., Azevedo, A. M., Karande, P., \& Cramer, S. (2019). Development of phage biopanning strategies to identify affinity peptide ligands for kappa light chain Fab fragments.Biotechnology Progress . https://doi.org/10.1002/btpr.2884

Nixon, A. E., Sexton, D. J., \& Ladner, R. C. (2014). Drugs derived from phage display: From candidate identification to clinical practice.MAbs , 6 (1), 73-85. https://doi.org/10.4161/mabs.27240

Sheth, R. D., Bhut, B. V., Jin, M., Li, Z., Chen, W., \& Cramer, S. M. (2014). Development of an ELP-Z based $\mathrm{mAb}$ affinity precipitation process using scaled-down filtration techniques. Journal of Biotechnology , 192 , 11-19. https://doi.org/10.1016/j.jbiotec.2014.09.020

Sheth, R. D., Jin, M., Bhut, B. V., Li, Z., Chen, W., \& Cramer, S. M. (2014). Affinity precipitation of a monoclonal antibody from an industrial harvest feedstock using an ELP-Z stimuli responsive biopolymer: Affinity Precipitation of a Monoclonal Antibody.Biotechnology and Bioengineering , 111 (8), 1595-1603. https://doi.org/10.1002/bit.25230

Sheth, R. D., Madan, B., Chen, W., \& Cramer, S. M. (2013). High-throughput screening for the development of a monoclonal antibody affinity precipitation step using ELP-z stimuli responsive biopolymers: HTS for Monoclonal Antibody Affinity Precipitation. Biotechnology and Bioengineering , 110 (10), 2664-2676. https://doi.org/10.1002/bit.24945

Shukla, D., Zamolo, L., Cavallotti, C., \& Trout, B. L. (2011). Understanding the Role of Arginine as an Eluent in Affinity Chromatography via Molecular Computations. The Journal of Physical Chemistry B , 115 (11), 2645-2654. https://doi.org/10.1021/jp111156z

Tsumoto, K., Ejima, D., Senczuk, A. M., Kita, Y., \& Arakawa, T. (2007). Effects of salts on proteinsurface interactions: Applications for column chromatography. Journal of Pharmaceutical Sciences ,96 (7), 1677-1690. https://doi.org/10.1002/jps.20821

Wu, J., Park, J. P., Dooley, K., Cropek, D. M., West, A. C., \& Banta, S. (2011). Rapid Development of New Protein Biosensors Utilizing Peptides Obtained via Phage Display. PLoS ONE , 6 (10), e24948. https://doi.org/10.1371/journal.pone.0024948

Yang, H., Gurgel, P. V., \& Carbonell, R. G. (2008). Hexamer peptide affinity resins that bind the Fc region of human immunoglobulin G: Fc-binding hexamer peptide resins. The Journal of Peptide Research , 66 , 120-137. https://doi.org/10.1111/j.1747-0285.2006.00342.x

Yeboah, A., Cohen, R. I., Rabolli, C., Yarmush, M. L., \& Berthiaume, F. (2016). Elastin-like polypeptides: A strategic fusion partner for biologics: Elastin-Like Polypeptides. Biotechnology and Bioengineering , 113 (8), 1617-1627. https://doi.org/10.1002/bit.25998

Table 1: Peptide sequences, along with their frequency of occurrence, obtained post 3 phage display biopanning rounds.

\begin{tabular}{lll}
\hline Peptide Sequence & Code & Frequency \\
H T T V H V R M P A M A & P1 & 4 \\
H F S F S Y K M P L S D & P2 & 6
\end{tabular}




\begin{tabular}{|c|c|c|}
\hline N V F H I T M P H D K H & $\mathrm{P} 3$ & 1 \\
\hline H G I A T A Y I R M P T & $\mathrm{P} 4$ & 1 \\
\hline H V W S W M W G N R Y A & $\mathrm{P} 5$ & 1 \\
\hline S S T L I W T G I K N T & $\mathrm{P} 6$ & 1 \\
\hline E P L S V F R F T M P W & P7 & 1 \\
\hline I S T A A W K D D Q R M & P8 & 1 \\
\hline H A W E F R V R L P A G & P9 & 1 \\
\hline D A L V W Q R A I T M P & P10 & 1 \\
\hline
\end{tabular}

Table 2: Binding dissociation constants $\left(\mathrm{K}_{\mathrm{d}}\right)$ of peptide candidates identified from phage display.

\begin{tabular}{ll}
\hline Peptide & $\mathbf{K}_{\boldsymbol{\delta}}(\mu \mathrm{M})$ \\
P1 & $>100$ \\
P2 & 58.2 \\
P4 & $>100$ \\
P7 & $>100$ \\
P9 & 109 \\
P10 & 19.5 \\
\hline
\end{tabular}

Figure legends:

Figure 1: Schematic of phage display technique carried out for peptide ligand discovery for AdP.

Figure 2: Fluorescence polarization binding curves for 6 peptides obtained by phage display against AdP with peptide P10 (inset) exhibiting the highest binding.

Figure 3: The binding of P10-ELP to AdP at various molar ratios of P10-ELP to AdP at pH 7.4.

Figure 4: (a) Binding of P10-ELP to AdP at different $\mathrm{pH}$ values $(3,4,5$ and 7.4) at a ratio of 2:1 of P10-ELP to AdP. (b) Binding of P10-ELP to AdP at various molar ratios of P10-ELP to AdP at pH 4.0.

Figure 5: (a) Effect of three different modifiers tested one at a time on the elution step recovery of AdP in pure component P10-ELP-AdP binding and precipitation experiments. (b) Effect of arginine concentration at different $\mathrm{pH}$ values on the elution step recovery of AdP.

Figure 6: SDS PAGE analysis of the purification of AdP from E. coli lysate.

Figure 7: C4-RP-UPLC chromatograms of AdP spiked into E. colilysate (top) and purified AdP after single step purification using P10-ELP (bottom).

\section{Hosted file}

Figures_Single Step Purification of a Small nonmAb Biologic.docx available at https: //authorea.com/users/317954/articles/447992-single-step-purification-of-a-small-nonmab-biologic-by-peptide-elp-based-affinity-precipitation 